\title{
SENTIMIENTOS Y PENSAMIENTOS DE JÓVENES FRENTE AL ACOSO ESCOLAR: EL SUICIDIO COMO UNA ALTERNATIVA*
}

\author{
FEELINGS AND THOUGHTS OF TEENAGERS IN THE FACE \\ OF SCHOOL BULLYING: SUICIDE AS AN ALTERNATIVE
}

\author{
Catalina Palacio-Chavarriaga** \\ LeIDY Johana RodRÍGuez-MaRín*** \\ Adriana María Gallego-Henao****
}

\section{Resumen}

Objetivo. Dar a conocer algunos sentimientos, pensamientos y visiones que tienen los estudiantes de noveno grado frente al tema del acoso escolar. Metodología. Se basó en el paradigma cualitativo desde un enfoque hermenéutico privilegiándose el estudio de caso como la estrategia de investigación. Participaron 35 adolescentes de una institución educativa de Medellín a quienes se les aplicaron 16 técnicas interactivas y ocho entrevistas semiestructuradas. Resultados. Se encontró que los jóvenes consideran el suicidio como alternativa para enfrentar el acoso escolar. Conclusión. Es importante sensibilizar a los maestros a no limitar sus acciones al momento de evidenciar conductas agresivas entre los estudiantes sino más bien preocuparse por desarrollar la empatía entre estos, de tal manera que sean capaces de ponerse en el lugar del otro.

Palabras clave: acoso escolar, suicidio, agresión, sentimientos, empatía.

\begin{abstract}
Objective. To introduce some feelings, thoughts and visions that ninth grade students have about bullying at school. Methodology. The methodology was based on the qualitative paradigm from a hermeneutic approach, privileging the case study as the research strategy. Sixteen interactive techniques and semi-structured interviews were applied to 35 teenagers from a school in Medellin. Results. It was found that young adolescents consider suicide as an alternative to face bullying. Conclusion. It is important to sensitize teachers to not limit their actions when they evidence aggressive behavior among students, but rather to develop empathy with them so that they can put themselves in the place of the other.

\footnotetext{
*Este artículo de investigación es resultado del proyecto denominado "Ciberacoso y empatía ¿cómo se concibe entre los jóvenes?", el cual se ejecutó entre 2017 y 2018. El producto se vincula al grupo de investigación Educación, Infancia y Lenguas Extranjeras.

${ }^{* * *}$ Universidad Católica Luis Amigó. Medellín-Colombia. E-mail: catalina.palacioch@amigo.edu.co.

(D) orcid.org/0000-0001-6201-2443. Google Scholar

**** Universidad Católica Luis Amigó. Medellín-Colombia. E-mail: leiidy.rodriguez29@gmail.com

(D) orcid.org/0000-0002-0064-643X. Google Scholar

***** Universidad Católica Luis Amigó. Medellín-Colombia. E-mail: adriana.gallegohe@amigo.edu.co.

(D) orcid.org/0000-0003-2952-1603. Google Scholar
}

rev. eleuthera. 21, julio-diciembre 2019, 34-47 
Key words: bullying, aggression, empathy, feelings, suicide.

\section{Introducción}

El suicidio ha sido una problemática vivida a lo largo de la historia humana, pero solo desde hace algunos años se vienen adelantando investigaciones relacionadas con el tema. Al respecto, la Organización Mundial de la Salud (2018) reportó que el suicidio es la segunda causa de muerte entre las personas cuyas edades oscilan entre los 15 y 29 años de edad, rango en el que se encuentran los participantes de la presente investigación.

Adicionalmente, en dicho informe se establece que los países con tasas mayores de suicidio son los de ingresos medios y bajos, estos según la clasificación del Banco Mundial (2018) son: Colombia, Bolivia, Guatemala, Costa Rica, Nueva Zelanda, entre otros. En este sentido, conforme a las estadísticas presentadas por Instituto Nacional de Salud (en adelante INS, 2014) Colombia ocupa el tercer puesto en la lista, antecedido solo por Cuba y Brasil respectivamente.

Según información del INS (2015) en el contexto colombiano, el suicidio se ubica en la cuarta posición en cuanto a las causas de muerte y son los hombres los que realizan más frecuentemente este tipo de actos, en una proporción de 1 a 4, es decir, por cada mujer que se quita la vida de manera intencional, cuatro hombres cometen el mismo acto; sin embargo, es importante aclarar que son más las mujeres que lo intentan, pero menos las que logran efectivamente este propósito.

Se destaca, además, conforme al informe técnico realizado por el INS (2015) que los departamentos con mayores tasas de suicidios son: Arauca, Vaupés, Amazonas y Putumayo; no obstante, es importante aclarar que en Antioquia, Bogotá y Valle del Cauca es donde se encuentra el mayor número de intentos de suicidio.

El Ministerio Nacional de Salud (MINSALUD) en su boletín No. 2 del 2017, establece que las causas más frecuentes por las cuales los jóvenes se suicidan están relacionadas con trastornos depresivos y afectivos ocasionados en los últimos tiempos por el bullying (Valadez, Amezcua, González, Montes y Vargas, 2011).

En este orden de ideas, se puede entender el acoso escolar como las conductas agresivas intencionadas que se dan dentro del contexto escolar entre los pares (Vessey como se citó en Villalobos, 2013). Sobre este tema se puede referenciar el estudio realizado en Costa Rica cuyo título es "El Bullying como posible factor de riesgo de conductas autolesivas suicidas en víctimas infantiles 
Sentimientos y pensamientos de jóvenes frente al acoso escolar: el suicidio como una alternativa

y adolescentes"(Villalobos, 2013). El autor resalta que el acoso escolar es un factor de riesgo que lleva especialmente a jóvenes a atentar contra su vida. Este estudio propuso tres estrategias para prevenir e intervenir situaciones de acoso escolar; estas son: a. Desarrollo de estrategias multimodales, es decir, que se lleve a cabo un trabajo mancomunado entre las diferentes dependencias de la institución (psicológico, pedagógico y administrativo); b. Elaborar políticas en el ámbito académico con el fin de prevenir conductas agresivas por parte de los estudiantes; y c. Sensibilizar a los docentes para que estén atentos a los cambios de comportamientos o conductas que presenten los estudiantes y lo reporte al equipo de apoyo.

Otro estudio de relevancia es el desarrollado en Montevideo, Uruguay, denominado El Bullying y su relación con el riesgo suicida en adolescentes. La población estuvo conformada por estudiantes de cuarto a sexto año de la educación básica (Freire, 2014). Los instrumentos utilizados fueron: la escala de agresión entre pares, subescala de influencia externa; de actitud hacia la violencia; de actitudes prosociales y de intimidación y pelea. Asimismo, se aplicó para evaluar el riesgo suicida el Inventario de Orientación Suicida. Estos instrumentos permitieron evidenciar como se ha abordado el tema y cuáles son los aspectos a tener en cuenta cuando se estudia el suicidio en relación con el bullying.

En el contexto colombiano, se encontró la investigación realizada por García, Moncada y Quintero (2013) titulada El Bullying y el suicidio en el escenario universitario, cuyo objetivo fue "presentar los resultados obtenidos del rastreo bibliográfico y documental que ha consistido en una revisión de las principales contribuciones de la literatura científica sobre el tema" (p. 2). Los resultados de este estudio demuestran que el bullying en el escenario universitario se manifiesta de manera diferente a la agresión física, es decir, se realiza maltrato psicológico por medio de expresiones verbales que tienen repercusiones igualmente devastadoras. Una de las conclusiones a la cual se llegó fue la necesidad de "implementar estrategias de prevención del suicidio con alcance en políticas públicas para evitar conductas suicidas en los jóvenes universitarios" (García et al., 2013, p. 309).

González, Mariaca y Arias (2014) en su investigación “Estudio exploratorio del bullying en Medellín” se trazaron como objetivo "realizar un muestreo exploratorio del fenómeno del bullying en Medellín, aportando indicadores de acoso escolar que tienen en cuenta los roles de las víctimas, los agresores y los testigos” (p. 20). Encontraron que el 48,17\% de los estudiantes han realizado bullying y a medida que avanzan en los grados escolares, dejan de realizar conductas agresivas. En cuanto a las víctimas, se halló que el 63,3\% han sufrido de acoso escolar y que estas personas se vuelven agresores posteriormente.

Adicionalmente, "se encontró que un $71,81 \%$ ha sido testigo de conductas violentas en el ámbito escolar” (González et al., 2014, p. 22), es así que la mayoría de los estudiantes que presencian estas conductas de agresión prefieren ocultar estas situaciones. De esta investigación 
se concluye que el bullying se realiza en presencia de muchas personas con el fin de captar la atención de los demás, igualmente si las víctimas y testigos deciden no manifestarlo, es probable que las agresiones permanezcan. Lo anterior evidencia que el acoso escolar no es desconocido por los jóvenes participantes del estudio, lo que indica que es necesario seguir estudiando esta problemática a profundidad con el fin de acompañar con mayor rigor a los adolescentes en su desarrollo integral.

\section{Metodología}

\section{Paradigma y enfoque}

El paradigma en el que se inscribe el estudio es el cualitativo (Martínez, 2004) asumiéndose el enfoque histórico hermenéutico desde el cual se permite entender la forma de pensar de las personas en un contexto determinado frente a una problemática específica (Gadamer, 1960).

\section{Participantes}

El escenario de este proyecto de investigación fue una institución pública que presta servicios educativos en Medellín. La muestra estuvo conformada por 35 estudiantes representados en $34 \%$ hombres y $66 \%$ mujeres. Los criterios de selección fueron: estar cursando noveno grado, tener una edad entre los 13 y 18 años, que usaran alguna red social, supieran de algún caso de acoso escolar y desearan hacer parte de este análisis. Es de anotar que después de socializar el proyecto con los estudiantes se procedió a explicarles que sus nombres nunca serían revelados y finalmente se procedió a firmar el consentimiento informado.

\section{Herramientas}

Los instrumentos utilizados para la recolección de información fueron 16 técnicas interactivas (árbol de problemas, mural de situaciones, retablo e historias que convocan) consideradas como actividades que posibilitan entender la forma en que se vivió una circunstancia en un contexto específico (García, González, Quiroz y Velásquez, 2003).

Adicionalmente, se realizaron ocho entrevistas semiestructuradas aplicadas a los estudiantes que durante las técnicas interactivas fueron participantes activos y expresaron conocer de cerca casos relacionados con acoso escolar o personas que acudieron al suicidio como forma de enfrentar dicha problemática. 
Sentimientos y pensamientos de jóvenes frente al acoso escolar: el suicidio como una alternativa

\section{Procedimiento}

Para iniciar la investigación, primero se habló con el rector de la Institución Educativa a fin de solicitar el permiso para llevar a cabo el estudio; una vez concedido, se socializó con los estudiantes de noveno grado la propuesta y se les explicó tanto el impacto de la misma como la importancia de su participación en esta. Posteriormente, se realizó la aplicación de los instrumentos y sistematización mediante un organizador gráfico que posibilitó unir información de cada una de las categorías y analizarlas conforme a la teoría encontrada en torno a ellas; luego se unificaron todos los cuadros de las investigadoras en una sola matriz. Finalmente, se redactaron los resultados que se detallan a continuación con relación a dos categorías del estudio: suicidio y acoso escolar.

\section{Resultados y discusión}

\section{Acoso escolar: visiones y perspectivas desde las voces de los adolescentes}

El acoso escolar según Olweus (como se citó en Chaux, 2013) es "la agresión repetida y sistemática que ejercen una o varias personas contra alguien que usualmente está en una posición de menos poder que sus agresores" (p. 126). En relación a esto, la Ley 1620 de 2013 refiere que el acoso escolar es una conducta sistemática que se repite en el tiempo, la cual puede ser psicológica o física y afecta la integridad del ser humano. Al respecto, los estudiantes que fueron víctimas o testigos de acoso escolar manifestaron sus comprensiones sobre el fenómeno de la siguiente manera: "El acoso es la agresión que una persona le hace a otra ya sea verbal o físicamente, así mismo cuando la persona es indefensa y se deja acosar de la otra persona" (Participante No. 11, comunicación personal, 20 de marzo de 2018). "Es molestar a alguien fuertemente y eso ya es muy delicado"(Participante No. 17, comunicación personal, 13 de marzo de 2018).

De lo anterior se puede señalar que sus comprensiones sobre el fenómeno del acoso escolar están relacionadas con las agresiones físicas y verbales que se hacen entre pares dentro de las escuelas. Esta concepción coincide con el trabajo investigativo realizado por González et al. (2014) al dar como resultado que: "La forma de agresión de mayor frecuencia es la verbal y la psicológica, y estas suceden usualmente en presencia de pares y docentes en el aula de clase” (p. 1). Allí se observa como la definición de acoso escolar no se encuentra muy alejada de las visiones o concepciones que tienen los participantes de otras investigaciones, las cuales concuerdan además con autores como Chaux (2013), quien lo define como la agresión física, verbal o indirecta que se hace de manera sistemática. Sin embargo, el acoso escolar es un fenómeno que no se puede simplificar y a la hora de estudiarlo a profundidad se deben tener en cuenta las características de la población y la cosmovisión que tienen los adolescentes de esta problemática. 
Dicho de este modo, los jóvenes participantes de este estudio coinciden en que el acoso escolar es una acción desagradable para quienes lo sufren y, más allá de esto, manifiestan que la persona afectada presenta emociones que pueden ser contraproducentes para su desarrollo personal y social como el odio, la tristeza y el rencor; así lo manifiesta uno de los participantes:

\begin{abstract}
A mí me acosaron, fue súper difícil y eso causó mucha desconfianza y depresión, ya no sé cómo hacer amigos, soy poco empática. Sinceramente cambié, porque valoraba mucho a los amigos, pero ya no, me encerré en mi propio mundo. (Participante No. 24, comunicación personal, 20 de marzo del 2018)
\end{abstract}

El relato permite inferir que los jóvenes, en su mayoría, prefieren ocultar lo sucedido y afrontar la situación con sus propios recursos emocionales, al respecto refiere González et al. (2014): "Lo que hace la víctima luego de haber recibido la agresión es, en primera instancia, no contarlo $(17,38 \%)$, decirle a un profesor $(15,5 \%)$, decirle a un amigo $(11,01 \%)$ o decirle a un familiar (10,54\%)" (p. 21). Estas cifras son un llamado de atención para maestros y cuidadores debido a que si los chicos callan esto puede conducir a un posible suicidio, en este orden de ideas es importante que se forme a los estudiantes en las diferentes alternativas que existen para detener estas situaciones, y así, prevenir las consecuencias que trae el acoso escolar.

Ceballos y Suárez (2013) refieren algunas de las causas y características que inducen al suicidio, entre ellas "la depresión, el aislamiento, la ansiedad, pérdida de apoyo por parte de sus seres queridos, inutilidad de la existencia, desesperanza, sentimientos de culpa” (p. 9). Estas causas y características la presentan las personas con tendencias suicidas, y pueden presentar afectaciones en sus estados emocionales por los que atraviesa diariamente; en este punto, su forma de verse en el mundo es diferente a la que comúnmente perciben otras personas que aprecian su existencia, es así como este agente suicida, al atravesar por determinadas circunstancias, puede tener como una alternativa el suicidio para terminar, definitivamente, con la difícil situación por la que pueda estar pasando.

En este orden de ideas, Blázquez (2000) menciona: "El suicidio podría ser una medida de emergencia para liberarnos de las miserias de esta vida cuando vemos que nos van faltando fuerzas para superarlas" (p. 354), de esta forma, el suicidio, en las situaciones del acoso escolar puede ser impulsado por las personas que constantemente están agrediendo a la víctima al generar presión sobre esta. A lo anterior ha de sumarse su incapacidad para defenderse adecuadamente.

Adicionalmente, Suárez y Osuna (2015) demuestran en su trabajo investigativo que tanto los padres como los docentes deben estar capacitados en habilidades sociales como: la solución de 
Sentimientos y pensamientos de jóvenes frente al acoso escolar: el suicidio como una alternativa

problemas, la empatía y el autocontrol, con el fin de crear programas de prevención del acoso escolar o realizar la intervención adecuada si este ya se presenta en el contexto escolar.

Los jóvenes manifiestan estar en la constante lucha de sobrevivir a pesar de la adversidad, en este caso, acudir al diálogo podría ser una alternativa para dar otro rumbo a su vida, sin tener que acudir a soluciones devastadoras, en este caso la autodestrucción. Asimismo, Frankl (1979) continúa diciendo que las maneras de afrontar las adversidades le sirven al individuo para llenar la vida de sentido, es decir, el buscar otras posibilidades para enfrentar el sufrimiento le permite al afectado mantener su dignidad y valorar su existencia, esto posibilita que las personas se fortalezcan en medio de los problemas y consigan "madurez interior" (Frankl, 1979, p. 93).

En suma, los maestros tienen la responsabilidad de generar espacios en las escuelas que les permitan a sus estudiantes desarrollar estrategias para comunicarse asertivamente y fomentar su pensamiento crítico (Chaux, 2013).

\section{Sentimientos que afloran en situación de acoso escolar: desde los relatos de los estudiantes}

Los sentimientos que emergen en los jóvenes de noveno grado a la hora de vivir situaciones de acoso escolar según Sandín (1967) se encuentran transversalizados por la experiencia previa que han vivido los sujetos, es decir, todas aquellas actividades que se desarrollan en los diferentes contextos donde interactúan, de tal manera que se les confiera un significado y se conviertan en sentimientos; siendo estas algunas de las formas como los jóvenes, en este caso particular, interpretan y se relacionan con su mundo.

El sufrir de acoso escolar puede tener diferentes reacciones y generar variados sentimientos en las víctimas. Algunos de estos, de acuerdo con lo expresado por los participantes en la investigación, son:

Me imagino que cuando a uno lo acosan siente ganas de suicidarse, yo creo que se le viene el mundo a uno encima porque uno no sabe qué hacer en ese momento, entonces es muy feo, debe sentirse uno lo peor. (Participante No. 33, comunicación personal, 20 de marzo de 2018)

Otro de los participantes manifiesta:

Se puede sentir dolor, preguntarse ¿por qué esto me pasa a mí?, ¿yo qué hice para merecerlo? Más que todos esos pensamientos antes pensabas, me voy a suicidar, pero eso es lo que más te pasa por la cabeza, y casi 
siempre se lo callan por sentir vergüenza o porque piensan, esto es mi problema como yo lo hacía antes, si yo no se lo hubiese contado a mi mamá yo estuviera súper mal ahora no estuviera aquí. (Participante No. 25, comunicación personal, 20 de marzo de 2018)

Lo dicho por los participantes permite inferir que al sufrirse de acoso escolar el suicidio es la posibilidad de huida de la situación; sin embargo, también se muestra una necesidad de ser escuchado y orientado por alguien de confianza quien podría ser un maestro o cuidador, una autoridad.

En este punto, Blázquez (2000) coincide con Chaux (2013) quien plantea la necesidad de desarrollar habilidades sociales en los estudiantes para enfrentar cualquier tipo de situación que represente un riesgo en la vida de las personas, sin ver como única salida el suicidio. No en vano existen los estándares básicos en competencias ciudadanas elaborados por el Ministerio de Educación Nacional de Colombia (MEN, 2006), con ellos se pretende contribuir al desarrollo de competencias que permitan a los estudiantes relacionarse asertivamente con sus pares y, de esta manera, logren resolver sus conflictos de manera pacífica.

Los participantes también mostraron su capacidad empática frente al tema al afirmar: "La víctima puede ser una persona depresiva, tener ganas de morirse, aislarse de la gente y llegar al suicidio" (Participante No. 17, comunicación personal, 13 de marzo de 2018).

Este fragmento permite inferir que el desarrollo de la empatía dentro del contexto escolar es una posibilidad acertada para prevenir conductas agresivas entre los compañeros de clase al ser un factor preventivo de dichos comportamientos. Además, a través de la empatía los individuos establecen relaciones sociales auténticas, enmarcadas en el respeto por el otro, por tanto, renunciarán a realizar conductas agresivas en los ambientes donde interactúan, al respecto señala Rincón (2011):

\footnotetext{
Hay un consenso general entre los especialistas de la educación acerca de que la empatía es uno de los elementos más importantes para prevenir y combatir la violencia entre los alumnos. La empatía es el elemento esencial de una comunicación auténtica que permite a los seres humanos sentirse verdaderamente acompañados y comprendidos. (p. 118)
}

En este orden de ideas, Chaux (2013) expresa que el desarrollo de la inteligencia emocional hace a las víctimas menos vulnerables ante cualquier tipo de ataques y las convierte en personas asertivas al momento de defenderse y romper con aquellos que puedan causarles daño. Igualmente, para los agresores el fomentar su empatía les permitirá verse a sí mismos en esa situación, lo cual conlleva a que los actos de violencia se vean disminuidos 
Sentimientos y pensamientos de jóvenes frente al acoso escolar: el suicidio como una alternativa

de manera significativa, al entender la situación por la que está pasando su víctima y sentir compasión por ella.

En las expresiones de los participantes se evidencia un elemento común en los relatos referidos a los sentimientos de depresión, tristeza, cambio de personalidad y baja autoestima, que puede llevar en los casos extremos a las víctimas a pensar en quitarse la vida, al creer que no existe otra solución para darle fin a su sufrimiento.

En este sentido, para Chaux (2013) es necesario que los maestros puedan plantear estrategias a fin de desarrollar competencias ciudadanas básicas en sus estudiantes para enfrentar la intimidación, estas son: la empatía cuando esta se genera, el agresor se detiene o elimina los deseos de hacer daño y de esta manera se reduciría significativamente las acciones de acoso escolar; la asertividad, la cual posibilita a la víctima reaccionar de forma correcta, cuando considere que no se le está dando el trato adecuado, y así detener a tiempo cualquier intento de acoso escolar; finalmente el pensamiento crítico, que le permite a los estudiantes reflexionar sobre sus conductas, es decir, pensar antes de actuar sobre las consecuencias de sus acciones.

En este aspecto, Olweus (2004) plantea que para manejar los casos de acoso escolar es necesario recurrir al diálogo, confrontando ambas partes, es decir, acosador y acosado, con el fin de lograr promover un ambiente de buen trato y disminuir la agresión por parte del victimario. Pikas (como se citó en Chaux, 2013), establece la necesidad de conversar a solas con la víctima, con el propósito de realizar un seguimiento y ayudarla a recuperar la confianza que ha perdido a causa de las agresiones recibidas.

Lo anterior está relacionado con una de las posibles soluciones referidas por los jóvenes participantes de este estudio, que es dialogar con las personas con las que tienen confianza y que les pueden ayudar. Aunque puede ser natural que en cierto momento sientan temor de expresar la situación por la que están pasando al hacerse a la idea de que nadie puede ayudarles y que la salida pueda ser recurrir al suicidio, no obstante, los adolescentes expresan que contarle su situación a otra persona ha servido para solucionar este tipo de conflictos.

En este sentido, Frankl (1979) al hablar de las adversidades por las que atraviesa el ser humano a lo largo de su vida establece que:

Las condiciones adversas le cierran al hombre las posibilidades de realizar valores de creación o de vivencia, pero aun así la vida continúa ofreciendo un sentido. En síntesis, cualquiera de los distintos aspectos de existencia conserva un valor significativo, el sufrimiento también. El realismo nos avisa de que el sufrimiento es una parte consustancial de la vida, como el destino y la muerte. Sin ellos, la existencia quedaría incompleta. (p. 92) 
Es decir, otra de las formas de afrontar el acoso escolar que emerge en los relatos de los adolescentes es contarle a los docentes o padres de familia un acto de agresión, a fin de conseguir apoyo entorno a la toma de decisiones en cuanto a las acciones que se han de seguir y así dar solución al problema. No obstante, en muchos de los casos las víctimas prefieren callar y afrontar por sí solos la situación.

En este sentido, se puede decir que en el acoso escolar se observa la fragilidad del ser humano al dejar una huella indeleble en la persona que lo sufre, especialmente en la víctima, quien generalmente tiende a callar lo que le pasa por temor a cualquier represalia por parte del acosador, muchas se encierran en su problema y su autoestima se ve vulnerada; esto las lleva a pensar que a nadie le puede interesar o preocupar su situación y quizás en algunos casos llegue a su mente la idea de atentar contra su vida como una solución a esta circunstancia.

Es normal que tengan la idea errada de que deben dar solución a las dificultades por su propia cuenta, por tanto, es vital darles a conocer las opciones que tienen a la hora de enfrentar esta problemática y hacerlos caer en cuenta de que un poco de ayuda no cae mal en estos casos. Asimismo, los docentes deben estar siempre prestos a escuchar a sus estudiantes, a fin de realizar un acompañamiento coherente a sus necesidades y así facilitar la superación de esta difícil situación.

En las voces de los jóvenes se evidencian los diferentes pensamientos que ellos tienen respecto a la forma de afrontar el acoso escolar al momento de estar inmersos en esta problemática, las dos reiterativas en varios relatos son: el aislarse de los compañeros de estudio y pensamientos suicidas al considerar que no cuentan con los recursos, para hacerle frente a la situación. En el estudio realizado por Carballo y Gómez (2017) se sugiere una mayor sensibilización a todos los agentes educativos (padres de familia, docentes, directivos y estudiantes) acerca de las consecuencias que trae el acoso escolar para quienes lo padecen. Se determina igualmente que "existe un acuerdo universal en afirmar la relación entre el bullying y la aparición posterior de ideas de suicidio” (Carballo y Gómez, 2017, p. 215).

Es decir, el fenómeno del suicidio se presenta en aquellas personas que atraviesan por diversas dificultades a lo largo de su vida y que, en ocasiones, se puede perder el control de estas situaciones al no regular su estilo de vida o por incapacidad de manejar de forma apropiada sus conflictos, lo cual genera desequilibrio e inestabilidad emocional, que lleva a las personas a presentar ideaciones suicidas. 
Sentimientos y pensamientos de jóvenes frente al acoso escolar: el suicidio como una alternativa

\section{Conclusiones}

El acoso escolar es un fenómeno que puede poner en aprietos a la persona que lo sufre, afectando significativamente sus estados emocionales, físicos y mentales, al estar expuestos constantemente al rechazo, burla, abandono, las cuales conllevan a conductas de profunda tristeza que pueden ser evidenciadas en diferentes comportamientos, entre ellos, la ansiedad, estrés, miedo y deseos de no seguir asistiendo al entorno educativo. Esto puede generar aislamiento, soledad y cerrarse a posibles alternativas que pueden solucionar su problema y, en los casos críticos, puede llevar al suicidio.

En este aspecto, el rastreo bibliográfico realizado por Villalobos (2013), arrojó como resultado que "la mayoría de investigaciones vincula al 'bullying' como un factor de riesgo relevante para que los niños y adolescentes víctimas presenten comportamientos autolesivos y/o suicidios" (p. 63). Debido a que este tipo de situaciones generan malestar a quienes son víctimas, llegan a considerar la posibilidad de atentar contra su vida como única solución a la dificultad por la que están atravesando.

Muchos de los jóvenes expresaron estar de acuerdo con los postulados anteriores al no encontrar el significado que tiene la vida en los momentos más adversos, es así como Frankl (1979) plantea el enfrentar la vida como una forma de encontrar la respuesta acertada a las diferentes cuestiones que la existencia presenta, en otras palabras, se trata de luchar por sobrevivir haciendo frente a los contratiempos que surgen a lo largo de la vida, uno de ellos puede ser el acoso escolar, de esta manera, los adolescentes, poco a poco, van construyendo su destino.

Para Frankl (1979) "cuando un hombre descubre que su destino es sufrir, ha de aceptar ese sufrimiento porque ese sufrimiento se convierte en su única y peculiar tarea” (p. 102), es decir, cada quien decide si aferrarse al sufrimiento que le provocó el acoso escolar, induciéndose al suicidio o superar victoriosamente esa situación por la que tuvo que atravesar al hacer uso de otras estrategias.

A raíz de lo anterior, se hace necesario que en el entorno educativo se den a conocer las rutas de atención a las cuales los estudiantes que son víctimas de acoso escolar pueden acudir, con el propósito de ponerle fin a dicha situación y, de esta forma, evitar las consecuencias que pueden llegar a ser tan graves como el acudir al suicidio para darle solución.

Así mismo, García et al. (2013) se basan en la investigación de Velarde y González (2011), los cuales concluyen que las ideas suicidas se presentan con mayor frecuencia cuando se sufre de acoso escolar. Cabe destacar que los jóvenes participantes de este estudio confirman lo que la literatura plantea respecto a la relación existente entre el suicidio y el acoso escolar, por lo 
cual es preciso trascender en las acciones de prevención no solo desde el manejo de la norma sino en la creación de programas para evitar el acoso escolar, con el fin de aprender a convivir pacíficamente con los demás.

La investigación de Villalobos (2013) sostiene que "algunas de las manifestaciones del bullying son aprendidas en el hogar o en la comunidad, incluso, influenciadas también por factores en el país y se desarrolla mediante un proceso de socialización culturalmente aceptado" (p. 55). Las conductas agresivas que ejerce el victimario pueden tener un origen desde el hogar y ser trasladados al contexto escolar, de esta manera Gómez (como se citó en González et al., 2014), dice que esta violencia puede pasar a ser un trato común entre algunos estudiantes y por tanto se evidencia como normal entre ellos, esto abre las puertas a posibles situaciones de violencia entre los estudiantes; por esta razón, se deben crear medidas de prevención y protección en los centros educativos debido a que los maestros, según Contreras (2013), no cuentan con la capacitación suficiente para detectar e intervenir en estas acciones.

En definitiva, se puede decir, que los participantes en su entorno escolar viven o evidencias situaciones de acoso escolar, no obstante, las personas víctimas de este flagelo generalmente no saben qué hacer en relación a este y optan por callar y sufrir en soledad y en algunos casos toman medidas extremas como el llegar a quitarse la vida. Por lo tanto, es necesario darles a conocer a todos los niños y adolescentes la ruta de atención integral que tienen las instituciones educativas para estos casos, con el propósito de ponerle fin a esta problemática y evitar consecuencias irreparables.

Se requiere asimismo realizar más investigaciones en las cuales se tenga en cuenta el punto de vista de los estudiantes que son víctimas y testigos de situaciones de acoso escolar, si se tiene en cuenta que son ellos quienes viven esta problemática socioeducativa de forma directa, por lo cual es pertinente hacer visibles sus voces con el propósito de desarrollar actividades contextualizadas para prevenirlas o superarlas.

\section{Referencias}

Banco Mundial. (2018). World Bank Country and Lending Groups. Country Classification. Recuperado de https:// datahelpdesk.worldbank.org/knowledgebase/articles/906519.

Blázquez, N. (Ed.). (2000). Bioética. La nueva ciencia de la vida. Madrid, España: Estudios y Ensayos BAC.

Carballo, J. y Gómez, J. (2017) Relación entre el bullying, autolesiones, ideación suicida e intentos autolíticos en niños y adolescentes. Revista Estudios de Juventud, 115, 207-2018. Recuperado de https: / / dialnet.unirioja.es/servlet/articulo?codigo $=6215272$. 
Sentimientos y pensamientos de jóvenes frente al acoso escolar: el suicidio como una alternativa

Ceballos, O. y Suárez, Y. (2013). El suicidio. Factores asociados por etapas evolutivas de la vida. Epidemiología del suicidio en el departamento de Magdalena y Santa Marta. Recuperado de https: / /books.google.com.co/books?isbn=9587460510.

Chaux, E. (Ed.). (2013). Educación, convivencia y agresión escolar. Bogotá, Colombia: Ediciones Uniandes.

Contreras, A. (2013). El fenómeno de bullying en Colombia. Revista Logos, Ciencia y Tecnología, 4 (2). Recuperado de http://revistalogos.policia.edu.co/index.php/rlct/article/ view/195/390.

Frankl, V. (Ed.). (1979). El hombre en busca de sentido. Barcelona, España: Ediciones Herder.

Freire, E. (2014). El Bullying y su relación con el riesgo suicida en adolescentes (tesis de pregrado). Universidad de la República Uruguay, Montevideo, Uruguay. Recuperado de https://sifp.psico.edu.uy/sites/default/files/Trabajos\%20finales/\%20Archivos/el_ bullying_y_su_relacion_con_el_riesgo_suicida._tfg_estefany_freire_1_0.pdf.

Gadamer, G. (1960). Verdad y Método. Recuperado de http://files.bereniceblanco1.webnode. es/200000089-633d56437f/-Gadamer-Hans-Georg-Verdad-y-Metodo-I.pdf.

García, B., González, S., Quiroz, A. y Velásquez, A. (2003). Técnicas interactivas para la investigación social cualitativa. Medellín, Colombia: FUNLAM.

García, J., Moncada, R. y Quintero, J. (2013). El bullying y el suicidio en el escenario universitario. Revista colombiana en ciencias sociales, 4 (2), 298-310. Recuperado de https:/ / dialnet.unirioja.es/descarga/articulo/5123784.pdf.

González, V., Mariaca, J. y Arias, J. (2014). Estudio exploratorio del bullying en Medellín. Pensando Psicología, 10 (17), 17-25. DOI: http://dx.doi.org/10.16925/pe.v10i17.776.

Instituto Nacional de Salud. (2015). Desigualdades Sociales en Salud en Colombia. Informe Técnico, Sexta edición. Recuperado de https://www.minsalud.gov.co/sites/rid/Lists/ BibliotecaDigital/RIDE/VS/ED/6to-informe-ons.pdf.

Instituto Nacional de Salud. (2014). Protocolo de vigilancia en salud pública, Intento de suicidio. Recuperado de https://www.minsalud.gov.co/sites/rid/Lists/BibliotecaDigital/ RIDE/IA/INS/protocolo-vigilancia-intento-suicidio.pdf.

Ley 1620 de 2013 (15 de marzo), por la cual se crea el Sistema Nacional de Convivencia Escolar y Formación para el Ejercicio de los Derechos Humanos, la Educación para la Sexualidad y la Prevención y Mitigación de la Violencia Escolar. Diario Oficial N. 48.733.

Martínez, M. (Ed.). (2004). Ciencia y arte en la metodología cualitativa. Ciudad de México, México: Editorial Trillas. 
Ministerio de Educación Nacional. (MEN 2006). Estándares Básicos de Competencias Ciudadanas en Lenguaje, Matemáticas y Ciudadanas. Recuperado de https://www.mineducacion.gov. co/1621/articles-340021_recurso_1.pdf.

Ministerio Nacional de Salud -MINSALUD-. (2017). Boletín de Salud Mental Conducta Suicida Subdirección de enfermedades no transmisibles. Boletín No. 2 julio. Recuperado de https: / /www.minsalud.gov.co/sites/rid/Lists/BibliotecaDigital/RIDE/VS/PP/ENT/ boletin-conducta-suicida.pdf.

Olweus, D. (Ed.). (2004). Conductas de acoso y amenazas entre escolares. Madrid, España: Ediciones Morata.

Organización Mundial de la Salud. (2018). Notas descriptivas, suicidio, Enero 2018. Recuperado de http://www.who.int/es/news-room/fact-sheets/detail/suicide.

Rincón, M. (Ed.). (2011). Bullying acoso escolar. Ciudad de México, México D.F.: Trillas.

Sandín, C. (Ed.). (1967). Pedagogía de las emociones. Alcoy, España: Marfil.

Suárez, G. y Osuna, N. (2015). Prevalencia del bullying relacional en agresores, agredidos y observadores en adolescentes escolarizados del municipio de Melgar-Tolima. Cultura, educación y sociedad, 6 (1). Recuperado de http://revistascientificas.cuc.edu.co/index. $\mathrm{php} / \mathrm{culturaeducacionysociedad/article/view/760.}$

Valadez, I., Amezcua, R., González, N., Montes, R. y Vargas, V. (2011). Maltrato entre iguales e intento suicida en sujetos adolescentes escolarizados. Revista en Ciencias Sociales, Niñez y Juventud, 9 (2), 783-796. Recuperado de http://www.redalyc.org/ pdf/773/77321592020.pdf.

Velarde, M. y González M. (2011). Los adolescentes que sufren de acoso escolar tienen más ideas suicidas. Recuperado de http://archivos.evidenciasenpediatria.es/files/41-11311RUTA/59AVC.pdf.

Villalobos, L. (2013). Bullying como posible factor de riesgo de conductas autolesivas o suicidas en víctimas infantiles y adolescentes (tesis de especialización). Universidad Rodrigo Facio, Ciudad de San José, Costa Rica. Recuperado de http://www.binasss.sa.cr/bibliotecas/ bhp/textos/tesis47.pdf. 